\title{
MEMS-in-the-lens architecture for a miniature high-NA laser scanning microscope
}

\author{
Tianbo Liu (1)', Milind Rajadhyaksha ${ }^{2}$ and David L. Dickensheets ${ }^{1}$
}

\begin{abstract}
Laser scanning microscopes can be miniaturized for in vivo imaging by substituting optical microelectromechanical system (MEMS) devices in place of larger components. The emergence of multifunctional active optical devices can support further miniaturization beyond direct component replacement because those active devices enable diffraction-limited performance using simpler optical system designs. In this paper, we propose a catadioptric microscope objective lens that features an integrated MEMS device for performing biaxial scanning, axial focus adjustment, and control of spherical aberration. The MEMS-in-the-lens architecture incorporates a reflective MEMS scanner between a low-numerical-aperture back lens group and an aplanatic hyperhemisphere front refractive element to support high-numerical-aperture imaging. We implemented this new optical system using a recently developed hybrid polymer/silicon MEMS three-dimensional scan mirror that features an annular aperture that allows it to be coaxially aligned within the objective lens without the need for a beam splitter. The optical performance of the active catadioptric system is simulated and imaging of hard targets and human cheek cells is demonstrated with a confocal microscope that is based on the new objective lens design.
\end{abstract}

\section{Introduction}

Scanning laser confocal and multiphoton microscopy techniques are a mainstay for in vivo imaging of unprepared, uncleared organs in live animals ${ }^{1-4}$. Substantial progress has been made in imaging small animals, such as mice, that can be immobilized on the stage of a benchtop microscope $\mathrm{e}^{5,6}$. Medical applications are also emerging. Large handheld or gantry-arm-mounted microscopes are used in dermatology clinics, which enable noninvasive and more thorough examination to reduce the dependence on physical biopsy for ruling out skin cancer $^{7-12}$. However, the large size of a conventional laser scanning microscope limits its potential for both medical and live animal imaging. For imaging ambulatory animals and for accessing most of the human body, miniaturization of these instruments is necessary.

\footnotetext{
Correspondence: David L. Dickensheets (davidd@montana.edu)

${ }^{1}$ Electrical and Computer Engineering Department, Montana State University, Bozeman, MT 59715, USA

${ }^{2}$ Dermatology Department, Memorial Sloan Kettering Cancer Center, New York, NY 10022, USA
}

Miniaturization of the scanning mechanism was a necessary first step in the development of smaller instruments. Microelectromechanical system (MEMS) devices replace the bulky mechanisms that are required for scanning and focusing the beam with components that are only millimeters in dimension. This has enabled applications that were not previously possible. For example, a MEMS-scanned miniaturized two-photon microscope that weighed only 2.15 grams and was small enough to be mounted on the head of a freely moving mouse was used to image neuronal dendrites and spines within the brain ${ }^{13,14}$. MEMS has also facilitated the adaptation of laser scanning microscopy to endoscopic platforms ${ }^{15-17}$ and MEMS-based optical biopsy systems have demonstrated in vivo detection of cancer in regions of the head, neck, esophagus, and cervix ${ }^{18-20}$.

In addition to having a small footprint, a MEMS scanner contributes to miniaturization by combining multiple degrees of freedom into a single active element. A biaxial MEMS scanner replaces two bulky galvanometer scanners and, potentially, a lens relay between them. A 3D MEMS 
scanner can realize focus control in addition to 2D lateral scanning via a tip/tilt/piston motion ${ }^{21-24}$, tip/tilt/curvature control ${ }^{25-27}$, or a combination ${ }^{28}$. This eliminates the need for motor-driven mechanical focusing and further reduces the instrument size.

However, the instrument size depends on the optical architecture, in addition to the optomechanical components. One choice that can influence the size is whether to use preobjective scanning or postobjective scanning. The majority of MEMS-scanned microscopes (and fiberscanned and fiber bundle systems) use preobjective scanning $^{29-36}$, which requires an objective lens that is well corrected over a finite field of view. Miniaturized, highnumerical-aperture lenses that have been developed for this configuration require multielement designs with tight tolerances and long optical paths, which affect the instrument $\operatorname{size}^{29,37-40}$. On the other hand, postobjective scanning requires an objective lens that is corrected only for axial performance, which could be a single, small aspheric element. However, the deployment of a scan mirror after the objective lens requires a long working distance and, therefore, yields the best results if a small numerical aperture is used, which is appropriate for optical coherence tomography or dual-axis confocal imaging ${ }^{41-46}$. For single-axis confocal or multiphoton microscopy, higher NA (typically $>0.7$ ) is required for adequate crosssectioning and signal strength ${ }^{47}$. For these applications, postobjective scanning is impractical for a small device.

A third alternative is to deploy the MEMS scanner within the objective lens. A simple back lens, which is only required to operate on axis, generates a converging beam of modest NA that is incident on the MEMS scanner. The scanned beam passes through an aplanatic front lens, which increases the NA in the sample while preserving the diffraction-limited imaging over a finite field of view. This architecture has been adopted for both dual-axis confocal $^{18,20,48,49}$ and fiber confocal fluorescence ${ }^{22}$ endoscopes with a hemisphere solid immersion lens as the front lens and tip/tilt/piston MEMS scanners. An aplanatic hemisphere increases the NA in the sample by $n$, which is the index of refraction of the glass; NA values of up to $\mathrm{NA}=0.38$ have been demonstrated ${ }^{22}$.

Here, we explore a new optical architecture for a miniature high-NA scanning laser microscope with a 3D MEMS scanner within the objective lens. We employ a folded annular beam, allowing the MEMS mirror to operate on axis. A low-NA back lens (only corrected on axis) illuminates the MEMS scanner. However, rather than a hemisphere solid immersion lens, we use an aplanatic hyperhemisphere front lens to increase the sample NA further. An aplanatic hyperhemisphere increases the NA by $n^{2}$, thereby allowing our instrument to operate with $\mathrm{NA}=0.7$. The $3 \mathrm{D}$ MEMS scan mirror that we employ is of the tip/tilt/curvature type, namely, it integrates focusing with a deformable mirror surface to control the wavefront curvature ${ }^{27}$. Compared to previous mirrors of this class ${ }^{25,26}$, this mirror is capable of at least 2.5 times the focus stroke and demonstrates a $\theta D$ product (a measure of the lateral resolution) of 12 deg-mm ( 0 to peak), which represents an improvement by a factor of 3.4 relative to the earlier mirror ${ }^{25}$. Furthermore, in this design, the 3D MEMS mirror has adaptive control for compensating spherical aberration throughout the 3D image volume, which becomes increasingly important for imaging at higher NA. The adaptive MEMS surface is a critical element in the overall objective lens design as it removes constraints on the glass elements and leads to a simple active optical system that preserves the diffractionlimited performance over a large 3D field of view.

The optical layout of the objective lens of the miniaturized confocal microscope is illustrated in Fig. 1a, b. By using an annular beam that passes through an aperture that surrounds the MEMS 3D scanner, the scan mirror can be integrated coaxially into the objective lens with the beam axis normal to the mirror surface without requiring a beam splitter to separate the incident and reflected beams. In our benchtop system (see Materials and Methods), we create the annular beam using a central stop, but it could be generated with greater optical efficiency by using, for example, axicon lenses or diffractive/ holographic optical elements. The beam is focused by the relatively low-NA back lens group. The converging beam passes through the annular aperture that surrounds the MEMS mirror and is reflected by a ring reflector onto the active surface of the 3D MEMS scanner. The converging beam is scanned by the MEMS device onto the hyperhemisphere front lens, which increases the NA. Lateral translation of the beam focus is accomplished by tip and tilt motions of the mirror. Axial translation of the beam focus is accomplished by changing the curvature of the MEMS mirror. Spherical aberration is managed through fine control of the shape of the MEMS mirror.

\section{Results \\ Simulation of the hyperhemisphere aplanat with active compensation of spherical aberration}

The aplanatic hyperhemisphere front lens is ubiquitous in high-NA oil immersion objective lenses. With oil immersion, the hyperhemisphere can be exact, with an object distance from the lens surface (including a layer of index-matched oil) equal to the lens radius times $1+1 / n$. At this depth, the spherical aberration and circular coma of all orders disappear ${ }^{50}$, leading to diffraction-limited imaging over a large field of view. Focusing is performed by translation of the object, which changes the thickness of the oil layer. When imaging beneath the surface, the spherical aberration and coma begin to increase if the sample and the oil differ in terms of index of refraction. 

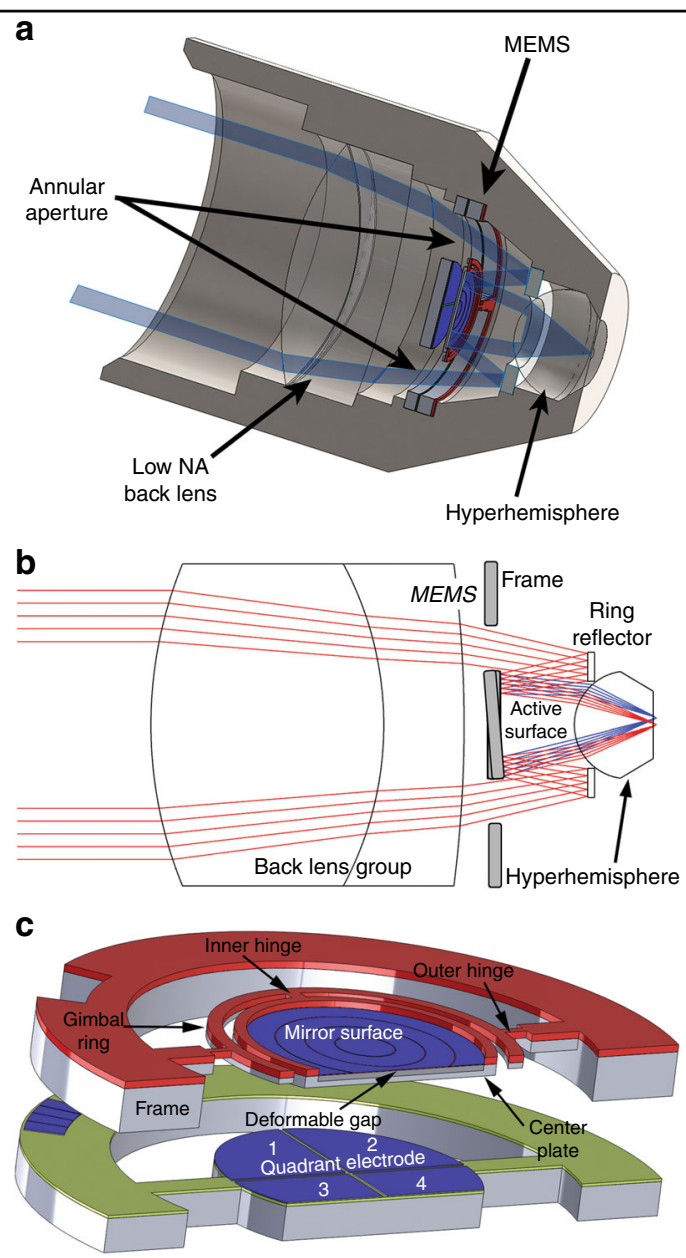

Fig. 1 MEMS-in-the-lens architecture. a A cross-sectional view of the miniaturized confocal microscope with a new objective lens that incorporates a MEMS 3D scanner. $\mathbf{b}$ An illustration of the light path through the annular aperture and the beam scan of the MEMS device. c A model of the MEMS 3D scanner. A gimbal platform is bonded to a set of quadrant electrodes

For in vivo microscopy, we wish to operate with the hyperhemisphere in contact with tissue, which has a variable index of refraction in a typical range of $1.3-1.4^{51,52}$. For our simulation, we assume $n$ for the tissue is constant with $n=1.34$, which is close to that of water. We can compensate the spherical aberration at a specified depth (e.g., $125 \mu \mathrm{m}$ ) beneath the surface of the tissue by using a slightly thinner hyperhemisphere lens. The solution is not strictly aplanatic, but it performs well over a sizable field of view. Figure 2 a shows the result of a full-aperture Zemax simulation for a $2 \mathrm{~mm}$ radius $\mathrm{BK}-7$ hyperhemisphere lens that is $3.151 \mathrm{~mm}$ thick at a depth of $125 \mu \mathrm{m}$ in the tissue. The NA is 0.7 , the aperture stop is located $2.5 \mathrm{~mm}$ in front of the lens surface (this would be the position of a scan mirror), and we plot the Strehl ratio as a function of the field of view. The simulation
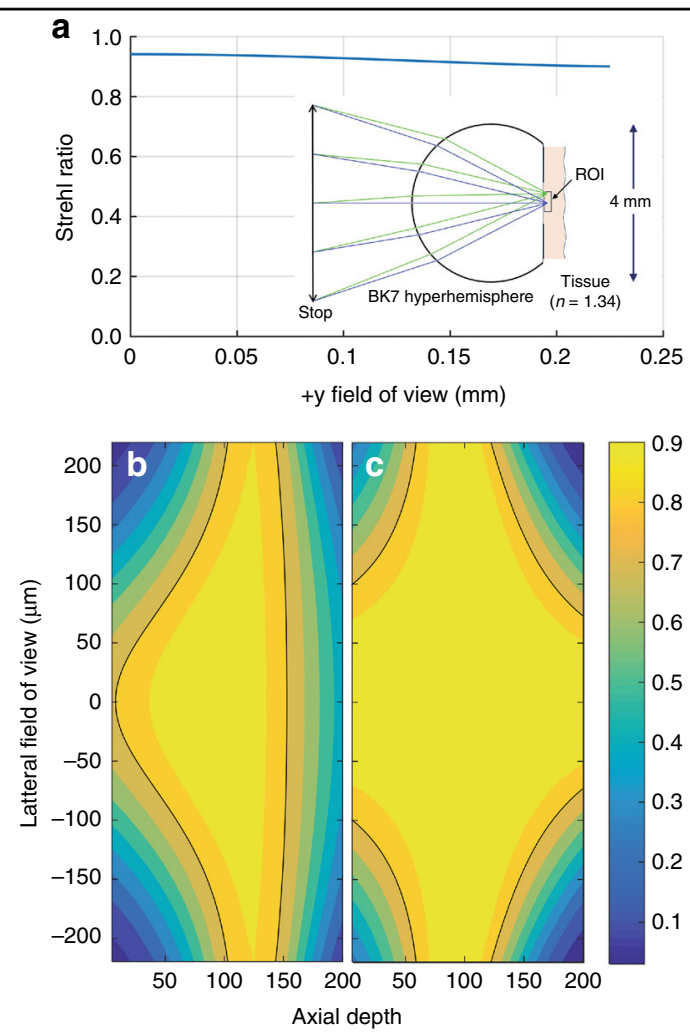

Fig. 2 Simulated imaging performance. a A Zemax simulation of a 2-mm-radius BK-7 hyperhemisphere that is in contact with tissue. The aperture stop is $2.5 \mathrm{~mm}$ to the left of the lens, with $\mathrm{NA}=0.7$. a A plot of the Strehl ratio vs. the lateral field, which was simulated for a depth of $125 \mu \mathrm{m}$. b, c A contour plot of the Strehl ratio over a 2D axial crosssection of the 3D field of view. The black line represents the contour for $S=0.8$. $\mathbf{b}$ Without depth-dependent adjustment of the spherical aberration. c With depth-dependent adjustment of the spherical aberration

wavelength is $633 \mathrm{~nm}$. At this depth, the system is diffraction limited over a lateral field of view of $>450 \mu \mathrm{m}$ (corresponding to a beam angle of \pm 2 degrees).

Figure $2 \mathrm{~b}$ presents a contour plot showing how the Strehl ratio varies over a depth of $0-200 \mu \mathrm{m}$ with a lateral field of view of $450 \mu \mathrm{m}$. In this plot, the wavefront curvature at the aperture stop is varied to control the focal depth in the tissue, with no control over the spherical aberration of the beam. The wavefront sag at the aperture stop changes by $41.4 \mu \mathrm{m}$ over the $200 \mu \mathrm{m}$ depth range. Hence, a variable mirror sag of $20.7 \mu \mathrm{m}$ is required to achieve a focus translation of $200 \mu \mathrm{m}$. According to this plot, the depth over which the lens is approximately aplanatic (and fully corrected over the full field of view) is fairly shallow. The dark contour corresponds to a Strehl ratio of 0.8 , above which the system can be considered diffraction-limited. According to this metric, $43 \%$ of the volume of the cylindrical $450 \times$ $200 \mu \mathrm{m} 3 \mathrm{D}$ field of view is diffraction-limited, with an average Strehl ratio over the full volume of 0.63. 
Figure 2c presents a similar contour plot of the Strehl ratio vs. the 3D field of view, except the spherical aberration is corrected at the aperture stop out to sixth order in the aperture radial variable, which provides a unique prescription for each depth in the simulation. This represents the effect of compensating the spherical aberration using dynamic surface shape control of the 3D MEMS mirror. In this case, the optimized hyperhemisphere thickness is $3.2 \mathrm{~mm}$. For the full range of the focus depth, the coefficient values for wavefront correction of the primary spherical aberration $\left(Z_{4}^{0}=\sqrt{5}\left(6 \rho^{4}-6 \rho^{2}+1\right)\right)$ ranged from $-38 \mathrm{~nm}$ to $+127 \mathrm{~nm}(-0.06$ to +0.2 waves at $633 \mathrm{~nm}$ ) and those for the secondary spherical aberration $\left(Z_{6}^{0}=\sqrt{7}\left(20 \rho^{6}-30 \rho^{4}+12 \rho^{2}-1\right)\right)$ ranged from +63 to $+253 \mathrm{~nm}(+0.1$ to +0.4 waves at $633 \mathrm{~nm})$ using normalized Zernike polynomials as the basis. The percentage of the 3D field of view that is diffraction limited has increased to $69 \%$, whereas the average Strehl ratio for the full field of view is 0.76 . From this simulation, we conclude that a simple hyperhemisphere of BK-7 glass can be an effective front lens element for a tissue microscope with NA $=0.7$ by using an active 3D MEMS scanner deployed at the location of the simulated aperture stop.

\section{Performance of the MEMS 3D beam scanner}

The 3D scanner (Fig. 1c) is based on a dual-axis gimbal platform suspended using polymer SU-8 hinges. The dual-axis architecture enables tip-tilt scanning, which is actuated by a set of quadrant electrodes placed underneath the gimbal. Integrated onto the center plate is a large-stroke deformable mirror for focus control. The focus electrodes are concentric and enable the control of spherical aberration. The diameter of the active optical surface is $4 \mathrm{~mm}$. An annular aperture is formed around the device to allow coaxial integration into the optical system. The mechanical properties of the mirror are summarized in Table $1^{27}$.

Applying the Rayleigh criterion, the resolution of a circular mirror can be expressed as ${ }^{53} N_{r}=4 \theta_{m} D / 1.22 \lambda_{o}$, where $N_{r}$ is the number of resolvable spots, $\theta_{m}$ is the zeroto-peak mechanical scan angle, $D$ is the diameter of the aperture $(4 \mathrm{~mm})$ and $\lambda_{o}$ is the imaging wavelength $(633 \mathrm{~nm})$. At the maximum measured resonant fast-axis

\section{Table 1 Mechanical performance of the MEMS 3D scanner}

\begin{tabular}{ll} 
Fast-axis resonant mechanical scan angle & $\pm 3^{\circ}$ \\
Fast-axis resonant frequency & $1000 \mathrm{~Hz}$ \\
Slow-axis resonant frequency & $\sim 200 \mathrm{~Hz}$ \\
Slow-axis $1 \mathrm{~Hz}$ mechanical scan angle. & $\pm 1.8^{\circ}$ \\
Maximum mirror sag. & $9.1 \mu \mathrm{m}$ \\
\hline
\end{tabular}

scan angle of $\pm 3^{\circ}\left(\theta_{m}=3^{\circ}\right)$, the mirror can resolve over 1080 spots. When operating at the $\pm 2^{\circ}$ mechanical scan angle $\left(\theta_{m}=2^{\circ}\right)$ used for the Zemax simulations in the previous section, the mirror can achieve a resolution of $N_{r}=726$ spots per line. The fast-axis resonant frequency of $1000 \mathrm{~Hz}$ allows imaging at four frames/second with a line density of 500 lines per frame using bidirectional scanning.

The range of axial focus is another crucial figure of merit for evaluating the performance of a confocal microscope. The mirror has demonstrated a deflection that exceeds $9 \mu \mathrm{m}$; when integrated into our optical system, this corresponds to $85 \mu \mathrm{m}$ of focus range $(N A=0.7$, $n=1.34)$. Previous membrane devices with similar construction have shown a $3 \mathrm{~dB}$ frequency response of $2 \mathrm{KHz}$ for focus control ${ }^{54}$. The deformable mirror has a measured correction range for $Z_{4}^{0}$ wavefront aberration from $-132 \mathrm{~nm}$ to $+228 \mathrm{~nm}(-0.21$ to +0.36 waves at $633 \mathrm{~nm})$ and $Z_{6}^{0}$ from $-178 \mathrm{~nm}$ to $+132 \mathrm{~nm}(-0.28$ to + 0.21 waves at $633 \mathrm{~nm}$ ). This range is sufficient for performing the full correction for primary spherical aberration $Z_{4}^{0}$ and a portion of the correction for secondary spherical aberration $Z_{6}^{0}$ to yield the result presented in Fig. 2c. If necessary, the glass optics can also be designed to partially compensate with a fixed correction that would shift the correction range to coincide with the range that is provided by the MEMS device. In that case, the fully corrected range specified in Fig. $2 \mathrm{c}$ would be available.

\section{Demonstration of confocal imaging}

Confocal imaging was demonstrated using a benchtop mock-up of the new objective lens with an integrated 3D MEMS mirror. A three-dimensional (3D) MEMS scanner was used to provide 2D imaging and focus control for the microscope. No active control of the spherical aberration was employed for these figures. In the demonstration system, the object-space NA is 0.57 , the pinhole NA is 0.06 , and the pinhole diameter is $10 \mu \mathrm{m}$. Figure 3a presents a confocal image of a portion of a prototype scan mirror, which clearly displays details such as the release vias (holes) on the surface of the aluminum. The mirror was attached to the sample stage using a thin layer of water-based ultrasound gel. The etch-release vias on the surface of the mirror are arranged in a $30 \mu \mathrm{m}$ square grid; the field of view of the system is $\sim 390 \mu \mathrm{m}$ by $180 \mu \mathrm{m}$. This corresponds to a $\pm 1.6^{\circ}$ mechanical angular scan along the fast axis $(y)$ and $\pm 0.75^{\circ}$ along the slow axis $(x)$. A digitally enlarged subsection of the mirror surface image is also displayed. The dimensions of the openings in the aluminum are $7 \mu \mathrm{m}$ by $7 \mu \mathrm{m}$. The dimensions of the holes that have been patterned into the underlying SU-8 membrane are $5 \mu \mathrm{m}$ by $5 \mu \mathrm{m}$. The scattered specks are imperfections on the mirror surface, which may have resulted from contamination during the deposition of the aluminum 


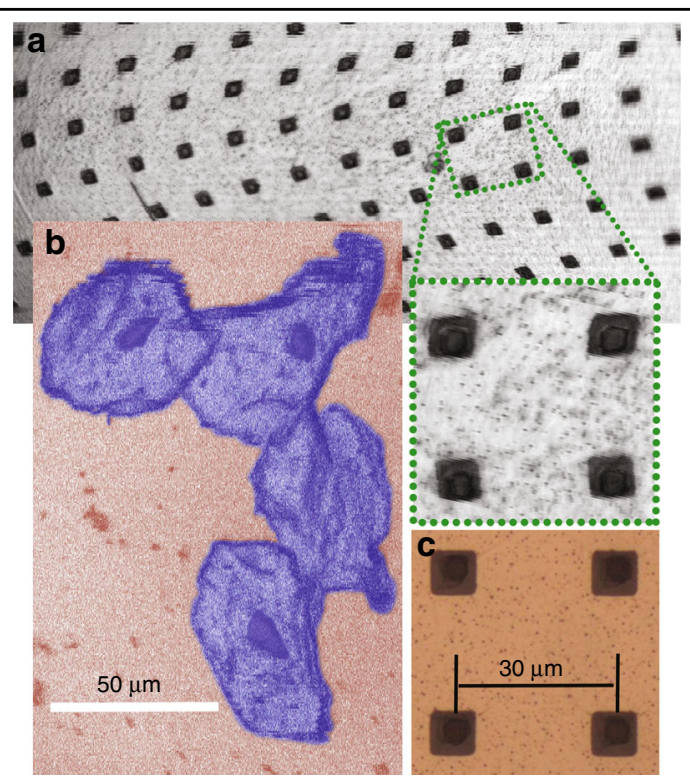

Fig. 3 Experimental imaging results. a A confocal image of the surface of a prototype three-dimensional scanner. A subsection of the image is digitally enlarged to show details. b A confocal image of human cheek cells (with false coloring). The nucleus and cell membranes are clearly visible. c A digitally cropped brightfield epiillumination microscope image of the surface of a similar prototype mirror that was recorded using a $\times 50$ objective lens $(N A=0.8)$

thin film. These specks are also visible under a brightfield, epi-illumination microscope $(\times 50$ magnification at $\mathrm{NA}=$ 0.8 ), as shown in Fig. 3c. The spatial arrangement of the vias follows a square grid pattern. However, according to the images, the linear grid appears to be slightly distorted. This could be owing to a misalignment of the electrodes underneath the gimbal, which pulls the mirror to one side at higher voltages and distorts the scan. This is more noticeable at the bottom of the frame, where the slow-axis voltage is the highest.

Figure 3b shows images of human cheek cells that were captured using the microscope. The cheek cells were introduced onto the sample stage using a cotton swab. A few drops of acetic acid ( $\sim 6 \%$ concentration) in the form of balsamic vinegar were applied to the cells to enhance the nuclear reflectance. (It has been suggested that acetic acid induces alterations in protein structure in the nucleus ${ }^{55,56}$.) The size of the cheek cells is $\sim 80 \mu \mathrm{m}$.

The axial sectioning capability of confocal microscopes allows imaging beneath the surface of the sample. The 3D scanner was used to demonstrate this. For this experiment, a sample composed of $6 \mu \mathrm{m}$-diameter polystyrene microbeads suspended in ultrasound transmission gel $\left(n_{\text {gel }}=1.3\right)$ was imaged. The initial focus of the system was positioned within the sample $(\sim 200 \mu \mathrm{m}$ axially $)$ so that the plane of imaging would remain in the sample as the beam focus was pulled toward the MEMS during the

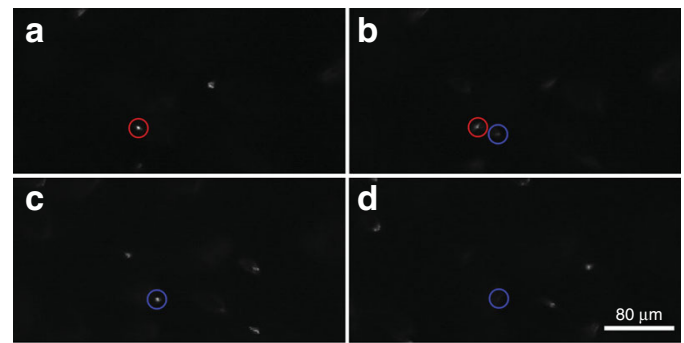

e

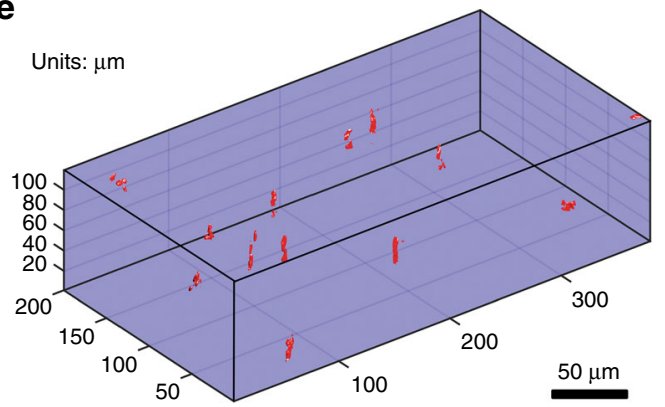

f

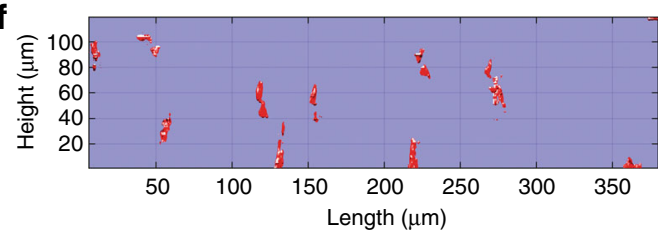

Fig. 4 3D imaging demonstration. a-d Confocal sectioning of 6$\mu \mathrm{m}$-diameter polystyrene beads suspended in ultrasound gel. Two beads have been circled using different colors to show their focus change from frame to frame. e A volumetric reconstruction from the images recorded at each focal plane. $\mathbf{f} A$ first-angle projection through the volumetric rendering to better illustrate the confocal sectioning at different focal planes

actuation of the deformable mirror. The applied focus voltage was from $0 \mathrm{~V}$ to $150 \mathrm{~V}$, with the same applied voltage on all four membrane electrodes. The focus shift in the sample varies approximately linearly with the square of the applied voltage. Nonuniform voltage steps were used to maintain a relatively consistent $z$-step size during the 3D image acquisition. The total voltagecontrolled focus range for the voltage range of $0-150 \mathrm{~V}$ was measured to be $127 \mu \mathrm{m}$ in the gel sample. Figure 4 displays en face images of the beads at four different focus locations. A $20 \mu \mathrm{m}$-diameter pinhole was used for this experiment to improve the signal-to-noise ratio. Images a-d are each separated axially by $26 \mu \mathrm{m}$. Axial sectioning is clearly observed, with the in-focus bead from frame (a) (red circle) blurred in frame (b) and, finally, not visible in frames (c) and (d). Figure 4e displays a volumetric reconstruction of the beads from the image stack acquired by the MEMS confocal microscope. The first-angle projection through the volume is shown in Fig. $4 \mathrm{f}$ to better illustrate confocal sectioning at different focal planes. The reconstructions in Fig. 4e, $\mathrm{f}$ display isosurfaces of the 
intensities of the bead objects that correspond to a threshold that is set at half of the peak intensity of the object (50\% intensity isosurfaces).

\section{Discussion}

We described a new objective lens architecture that positions a 3D beam scanner between a high-NA aplanatic hyperhemisphere front element and a lower NA back lens group. The instrument features an annular aperture, allowing the scan mirror to be integrated into the objective lens, coaxially aligned to and normal to the optical axis without a beam splitter to separate the reflected beam from the incident beam. The back lens group can be a simple asphere as it needs only be well corrected for on axis spherical aberration (and, possibly, to provide chromatic correction for a fluorescence microscope). If the front lens is aplanatic (corrected for spherical and coma aberrations), then the performance of the integrated system can be diffraction limited over a wide field of view at depths where the aberration compensation is maintained.

The simulated performance of a $2 \mathrm{~mm}$ radius BK-7 hyperhemisphere aplanatic lens showed that the lens could be diffraction limited over $43 \%$ of a cylindrical 3D sample volume that was $450 \mu \mathrm{m}$ in diameter and $200 \mu \mathrm{m}$ deep, with the scanner only controlling the beam tip, tilt, and focus. However, with adaptive control over the spherical aberration, the imaging can be diffraction limited over $60 \%$ more volume, which corresponds to up to $69 \%$ of the full 3D sample volume. On axis, the correction is nearly perfect, and diffraction-limited performance was observed laterally over a finite field of view of $150 \mu \mathrm{m}$ up to $200 \mu \mathrm{m}$ depth. This will be useful for depth-resolved imaging for in vivo microscopy, for example.

Simulations were performed using a wavelength of $633 \mathrm{~nm}$. For an optical pathlength aberration, the Strehl ratio improves for longer wavelengths because the optical pathlength error becomes less significant relative to the longer wavelength. On the other hand, using shorter illumination wavelengths, for example, for one-photon fluorescence excitation, the diffraction-limited volume will become slightly smaller, but the variation will be small across the visible spectrum. According to Maréchal's formula, an RMS wavefront error resulting in a Strehl ratio of 0.9 at $633 \mathrm{~nm}$ would correspond to a Strehl ratio of 0.8 (and, therefore, still be considered "diffraction limited") at $441 \mathrm{~nm}$. At shorter wavelengths, light penetration into the tissue is diminished because of scattering, especially in heavily scattering tissues such as skin. In such cases, the system can be optimized for operation at shallower depths. On the other hand, at longer wavelengths, where there is less tissue scattering and the penetration depths can be higher, the fully corrected volume will be somewhat larger. For fluorescence microscopy, which requires correction at both the excitation and emission wavelengths, the back lens group could provide chromatic correction.

We developed a 3D MEMS mirror scanner that provides complete scanning and focus control for the instrument while also providing electronic control of the spherical aberration. The new 3D scan mirror demonstrates an improvement in the focus control range of $2.5 \times$ and an improvement in the lateral scan resolution of $3.4 \times$ compared with previously described 3D MEMS mirrors $^{25,26}$. The mirror is provided with an annular aperture to allow its incorporation into a compact MEMS-in-thelens system.

Finally, we built a mock-up of the proposed confocal system using our 3D MEMS scanner. We successfully used it to demonstrate imaging of a structured, highreflectivity film, a sample of human cheek cells and confocal sectioning of suspended polystyrene beads. We clearly resolved submicron features in the highly reflective sample and showed confocal cross-sectioning when imaging the matrix of suspended beads. In the front projection image in Fig. 4f, whereas the beads are clearly resolved, the 3D profiles of each bead are neither uniform nor symmetric. This is a consequence of unavoidable interference effects (these are reflected light images, not fluorescence images) created by weak back-reflections from various uncoated glass surfaces; the symmetry may be further degraded by a small amount of astigmatism that was inadvertently introduced onto the MEMS scanner during manufacturing. Additional details are provided in the Materials and Methods section.

We have proposed a catadioptric MEMS-in-the-lens microscope objective lens that features an integrated MEMS 3D scanner for performing biaxial scanning and axial focus adjustment with dynamic control of spherical aberration. Based on our investigation, we believe the proposed instrument architecture shows considerable promise for future miniaturized high-NA laser scanning microscopes for in vivo imaging.

\section{Materials and methods}

\section{Fabrication of the MEMS 3D scanner}

The 3D scanner was constructed by bonding a micromachined gimbal platform with an integrated deformable mirror to a set of quadrant electrodes for tip-tilt actuation. The gimbal structure was fabricated with a silicon on insulator (SOI) wafer, whereas the electrode portion was fabricated using a double-side-polished silicon wafer. These two wafers will be referred to as the gimbal wafer and the electrode wafer, respectively. The gimbal platform features a silicon center plate that is supported by an outer silicon gimbal ring that is suspended via SU-8-based torsional hinges. Integrated onto the center plate is a deformable mirror that can be actuated using its own set 


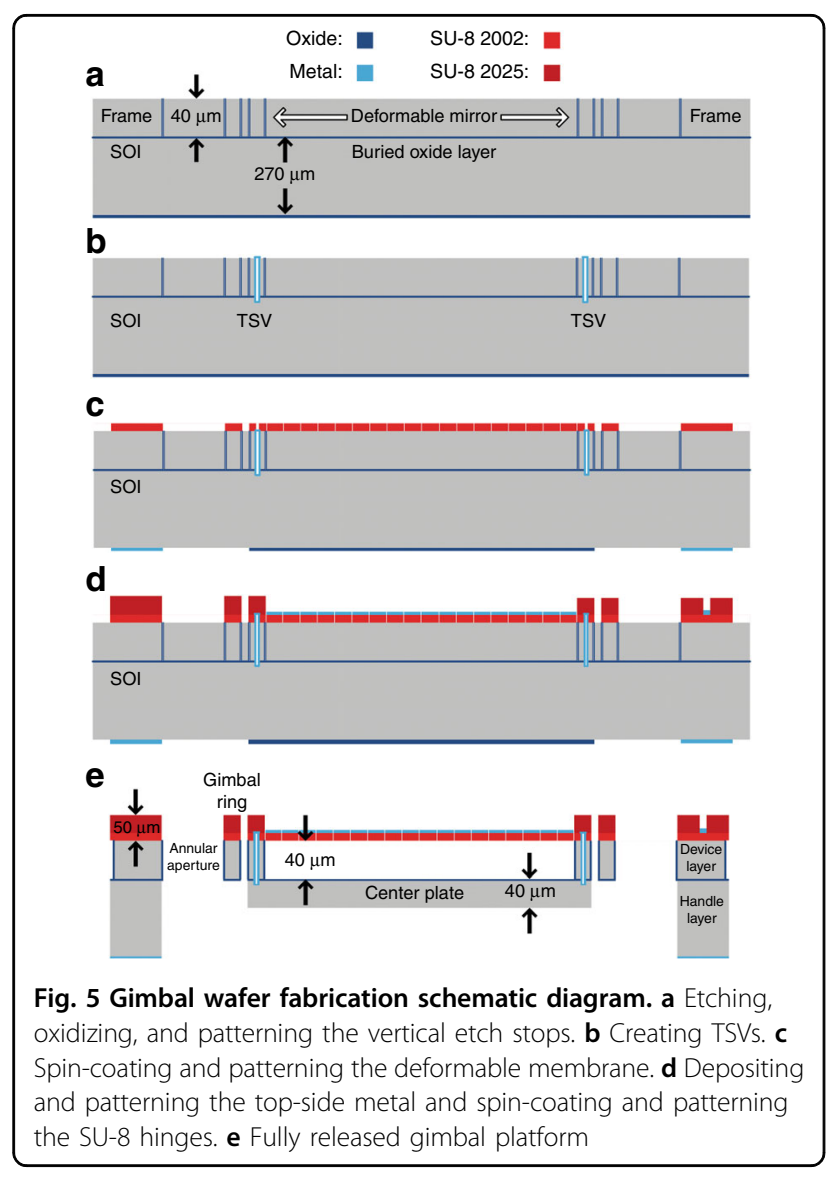

of electrodes. The electrode wafer is of simpler construction: it carries a set of quadrant electrodes encapsulated in a dielectric polymer to prevent shorting in the event of incidental physical contact with the upper structure.

The fabrication process was described in detail by Liu et $\mathrm{al}^{27}$. An overview of the process is provided here. The SOI gimbal wafer consists of device, handle and buried oxide layers with thicknesses $40 \mu \mathrm{m}, 270 \mu \mathrm{m}$, and $300 \mathrm{~nm}$, respectively. Figure 5 presents a schematic diagram of the fabrication process. Fabrication begins with vertical oxidized etch stops that are used during the release process to accurately define the dimensions of critical features. These etch stops were created by etching $3 \mu \mathrm{m}$ wide trenches through the device-layer silicon to define the dimensions of the released features. Then, the wafer was oxidized and patterned to form the etch stops. The oxide on the backside of the wafer was also patterned during this step to create the first part of a bilayer differential etch mask that was used during the release step to define the thickness of the center gimbal plate. Next, throughsilicon vias (TSVs) were formed to allow electrical connection from the surface of the SOI wafer to the handlelayer silicon, which would eventually become the center plate and act as the ground electrode for both scanning and varifocal actuation. To do this, vias were etched from the device-layer silicon past the buried oxide and into the handle-layer silicon. Then, they were coated with metal to provide electrical connection. The successful fabrication of these vias relied on a new and simple technique that uses progressive via sizing to mitigate the notching at the silicon-to-buried-oxide interface ${ }^{27,57}$. After the completion of the TSVs, aluminum was evaporated onto the backside of the wafer and patterned to form the second part of the bilayer differential etch mask. Next, the deformable membrane was constructed by spin-coating and patterning a $4 \mu \mathrm{m}$ thick layer of SU-8. During this step, release vias on the surface of the membrane and ports were also formed to allow access to the TSVs. Then, a liftoff process was used to pattern a thin layer of aluminum $(100 \mathrm{~nm})$ as the optical surface of the deformable mirror. This reflective metallic surface was partitioned into four concentric rings to serve as the electrodes for varifocal actuation. The electrical bond pads on the frame of the device and the metal traces that route the electrical connection to the TSVs and concentric electrodes were also formed during this step. Then, thick polymer hinges and hinge anchors were formed by spin-coating and patterning a $46 \mu \mathrm{m}$ thick layer of SU-8 2025. This layer, along with the previous $4 \mu \mathrm{m}$ layer of thin SU-8, fulfills the designed thickness requirement of $50 \mu \mathrm{m}$ for the SU-8 flexures.

The release process begins with the use of the differential etch mask on the backside of the wafer to roughly define the thickness of the center plate. A silicon dry etch was used to create a step differential of $\sim 50 \mu \mathrm{m}$. Then, dry oxide etching was performed to remove the oxide that covered the center gimbal plate. Next, the silicon etch was employed again to within $10 \mu \mathrm{m}$ to $20 \mu \mathrm{m}$ of the buried oxide. This remaining silicon was retained temporarily for structural integrity during subsequent steps. Then, xenon difluoride was applied to the top side only to remove the silicon from underneath the deformable mirror, thereby leaving it free-standing above the center plate. The silicon from between the gimbal structures and within the annular aperture was etched away simultaneously during this step. This xenon difluoride etch, although isotropic by nature, becomes guided and confined through the combined effects of the oxidized vertical etch stops, the buried oxide layer and the thin SU-8 film. Next, xenon difluoride was used to etch the backside of the wafer to clear the remaining silicon below the buried oxide. As the final step, a low-power dry oxide etch was used from the backside of the wafer to remove the exposed buried oxide. Figure 6a shows the devices on the wafer after the release process.

The fabrication of the electrode wafer was simpler. A double-side-polished wafer was oxidized, metallized, and 


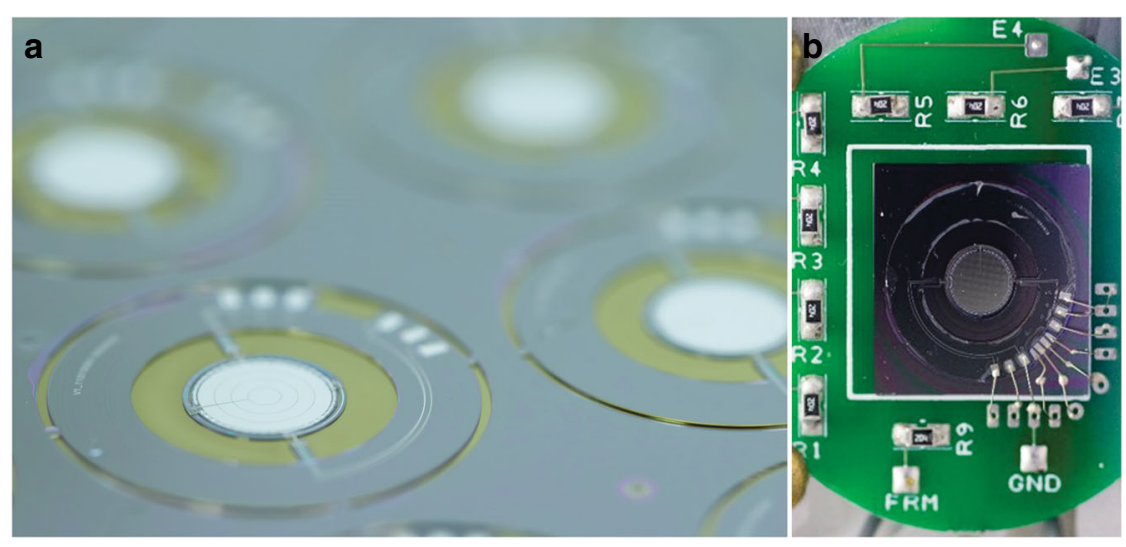

Fig. 6 3D MEMS scan mirror. a Devices on wafer after the release process. $\mathbf{b}$ The MEMS scanner after wirebonding to support PCB

patterned to form a set of quadrant electrodes and bond pads for electrical connection to external supporting printed circuit boards (PCBs). The design and fabrication processes of the electrode wafer accommodate the option of an annular aperture matching that of the gimbal platform. As this aperture does not affect the imaging results in this paper, it was omitted for ease of fabrication. A layer of SU-8 was spin-coated onto the electrode wafer to prevent shorting of the center plate to the quadrant electrodes in the unpredicted event of physical contact. Then, the singulated devices from the gimbal wafer and the electrode wafer were aligned and bonded. The completed 3D scan mirror was wire-bonded to a supporting PCB, which is shown in Fig. 6b.

\section{Focus depth and spherical aberration control}

The 3D MEMS scanner adjusts the focus by electrostatically changing the curvature of the optical surface. The axial Rayleigh resolution, defined as the distance from the peak of the axial light distribution to the first null, can be expressed as $z_{R}=2 n \lambda_{o} / N A^{2}$, where $n$ is the index of refraction of the medium being imaged (human skin: $n=$ $\left.1.34^{51,52}\right), \lambda_{o}$ is the vacuum wavelength, and NA is the image-space numerical aperture. For the miniaturized microscope described in this paper, for NA $=0.70$ and $\lambda_{o}$ $=633 \mathrm{~nm}, Z_{R}=3.46 \mu \mathrm{m}$. Based on paraxial Fourier analysis of a circular pupil, the phase delay that is required for shifting the focus by $z_{R}$ is $2 \pi \rho^{2}$, where $\rho$ is a normalized radial variable at the pupil. A mirror sag of $\lambda_{o} / 2$ is necessary to achieve this phase delay. Therefore, the number of axial zones (Rayleigh distances) that are resolvable can be expressed as $N_{z}=2 \delta / \lambda_{o}$, where $\delta$ is the maximum achievable mirror deflection. At higher NA, a derating factor $a$ should be included such that $N_{z}=2 a \delta / \lambda_{o}$, with $a=0.86$ for NA $=0.7$. In this paper, the mirrors have demonstrated deflections that exceed $9 \mu \mathrm{m}$, which, when integrated into our optical system, corresponds to $N_{z}=2 \times 0.86 \times 9 / 0.633=24.5$ resolvable zones. This provides $85 \mu \mathrm{m}$ of focus range. This maximum deflection is currently limited by electrostatic pull-in. For our next generation of mirrors, a larger air gap under the membrane can further increase this focus range.

As described in the Results section, the spherical aberration of the system changes as a function of the focus depth. Therefore, the mirror was designed with concentric electrodes that add an additional degree of freedom for further tuning the optical surface to offset the induced spherical aberration as it is defocused. To vary the force radially on the deformable mirror, the concentric electrodes are biased using independent voltages. The amount of spherical aberration correction that can be achieved at a given defocus is limited by the differential voltage that can be tolerated between the electrodes, which is limited by electrical breakdown (arcing). The location on the 3D scanner that is most prone to electrical breakdown is at the hinges, where the separation between neighboring electrode traces becomes as small as $8 \mu \mathrm{m}$. To avoid arcing, it is necessary to evaluate the maximum voltage differential that can be tolerated. To do this, each of the concentric electrodes, in turn, were biased relative to the remaining three electrodes. The experimental results showed that the electrodes can handle differential voltages in excess of $200 \mathrm{~V}$ and up to $250 \mathrm{~V}$ between the outermost electrode and the innermost electrode. The increased tolerance between the outermost and innermost electrodes is owing to the layout of the electrodes and the traces on the device. Adhering to the voltage limitations that are specified above, the ranges of adjustment for firstorder $\left(Z_{4}^{0}\right)$ and second-order $\left(Z_{6}^{0}\right)$ spherical aberration (coefficients of the normalized Zernike polynomials) were evaluated, which were superimposed on a baseline defocus $\left(Z_{2}^{0}\right)$ value of $\sim 1068 \mathrm{~nm}(3.7 \mu \mathrm{m}$ nominal deflection). The range of observed values for the normalized Zernike coefficient of $Z_{4}^{0}$ was $-66 \mathrm{~nm}$ to $+114 \mathrm{~nm}$ for the mirror 


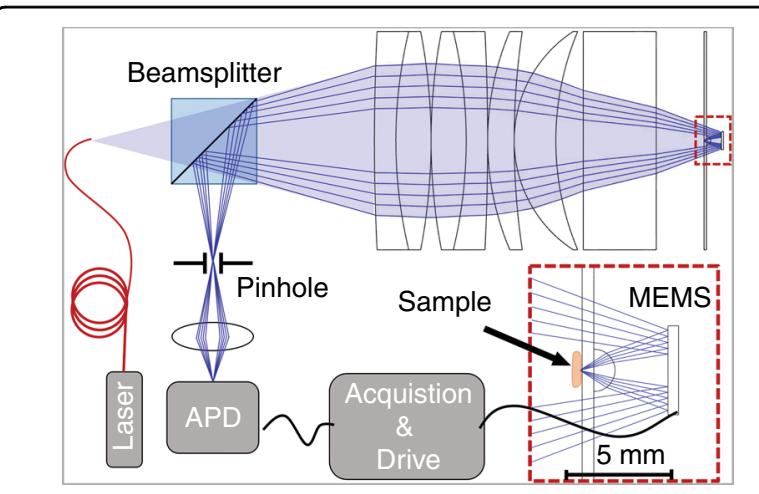

Fig. 7 Schematic diagram of the confocal imaging setup. A magnified view of the MEMS scanner, hyperhemisphere, and sample stage is also included

surface height, which corresponds to $-132 \mathrm{~nm}$ to + $228 \mathrm{~nm}$ of wavefront aberration. The range of observed values for the normalized Zernike coefficient of $Z_{6}^{0}$ was $89 \mathrm{~nm}$ to $+66 \mathrm{~nm}$ for the mirror surface height, which corresponds to $-178 \mathrm{~nm}$ to $+132 \mathrm{~nm}$ of wavefront aberration. This does not represent the full range of values that can be achieved. A more in-depth analysis of the spherical aberration adjustment performance that can be realized using a deformable mirror that is similar to that of this paper was conducted by Lukes et al. ${ }^{58}$.

\section{Benchtop imaging demonstration}

A confocal microscope was constructed for assessing the imaging performance of the new objective lens with an integrated 3D MEMS mirror. A schematic diagram of the optical setup is shown in Fig. 7.

The benchtop system follows the compact optical system diagramed in Fig. 1b, except without the retroreflection from an annular ring mirror. The beam is only reflected by the MEMS scanner, which can now be conveniently mounted at the right side of the setup. The system preserves the essential order with the sample, imaged by a hyperhemisphere lens, followed by the active 3D mirror scanner, as illustrated in the inset of Fig. 7. It also employs an annular beam. The observed performance is fully representative of the proposed MEMS-in-the-lens architecture. A consequence of the simpler optical test setup is that the sample is now located within the system, where it must be placed in contact with the glass using gel; the sample must also not exceed the diameter of the hyperhemisphere lens to prevent further obscuration of the imaging beam. However, this did not limit the field of view and this method of sample mounting did not negatively impact our experiments.

The illumination was from a $633 \mathrm{~nm}$ helium neon laser. The optical fiber was a single-mode fiber with NA between 0.10 and 0.14 and a mode field diameter of
3.6-5.3 $\mu \mathrm{m}$ (Thorlabs SM600 fiber). The objective lens system had an effective focal length of $14.78 \mathrm{~mm}$ (in air), an image-space NA of 0.57 and an object-space NA of 0.06, which was limited by the MEMS mirror as the aperture stop. The back compound lens group is also illustrated in Fig. 7. This lens group comprises two backto-back achromatic doublets (Thorlabs AC508-200, $f=$ $200 \mathrm{~mm}$ ) followed by two meniscus lenses (Thorlabs LE1015, $f=200 \mathrm{~mm}$ and Thorlabs LE1076, $f=100 \mathrm{~mm}$ ), all of which are in contact. A thick glass plate $(18 \mathrm{~mm})$ provides spherical aberration compensation for the desired focus depth of the instrument. A $2 \mathrm{~mm}$ diameter hyperhemisphere front lens was constructed from a $2 \mathrm{~mm}$ diameter half-ball lens (BK-7 glass) centered on and cemented to a $500 \mu \mathrm{m}$ thick, $50.8 \mathrm{~mm}$ diameter glass wafer (D263T ECO glass), which also serves as the sample stage. The sample was attached to the side of the glass wafer opposite to the hyperhemisphere lens. The resultant $1.5 \mathrm{~mm}$ thick glass hyperhemisphere with $1 \mathrm{~mm}$ radius of curvature has minimal spherical aberration when imaging at a depth of $110 \mu \mathrm{m}$ in water.

The MEMS scanner was mounted onto a stage (not shown) with three degrees of translational freedom and two degrees of rotational freedom facilitating focus adjustment and alignment. A 50/50 beam splitter was situated between the optical fiber and the compound lens element to separate the reflected light. A $10 \mu \mathrm{m}$-diameter pinhole was positioned conjugate to the optical fiber to spatially filter the reflected light. An avalanche photodiode detector was used to collect the light. The image forming beam is an annular beam, with the central portion blocked by the hyperhemisphere lens and the sample during the forward passage through the transparent sample stage. During imaging, a raster scan pattern was used in which the slow axis was driven nonresonantly using a sawtooth waveform $\left(V_{y}\right)$, whereas the fast axis $\left(V_{x}\right)$ was driven at its resonant frequency using a sinusoidal waveform. To date, the polymer flexures have shown no effects of aging (no change in the resonant frequency) despite accumulating over one billion cycles for the fast axis $(>280 \mathrm{~h}$ of operation).

The control voltages that were applied to the quadrant electrodes (Fig. 1b) are as follows: $V_{1}=V_{\mathrm{DC}}+V_{x}+V_{y}$, $V_{2}=V_{\mathrm{DC}}-V_{x}+V_{y}, V_{3}=V_{\mathrm{DC}}-V_{x}-V_{y}$ and $V_{4}=V_{\mathrm{DC}}$ $+V_{x}-V_{y}$. For Figs. 3a, b, 4, the applied voltages were $V_{D C}=300 \mathrm{~V}, V_{x}=200 \mathrm{~V} \mathrm{pk-pk}$ and $V_{y}=300 \mathrm{~V} \mathrm{pk}-\mathrm{pk}$. Figure 3 was cropped for display. Linear interpolation has been applied to all of the confocal images that are displayed in the Results section to correct for the sinusoidal distortion of the fast scan.

The edge response was measured by using the MEMS scanner to image the edge of a cleaved wafer piece. A $65 \mu \mathrm{m}$ thick microscope coverslip was inserted between the wafer piece and the sample stage to place the wafer 

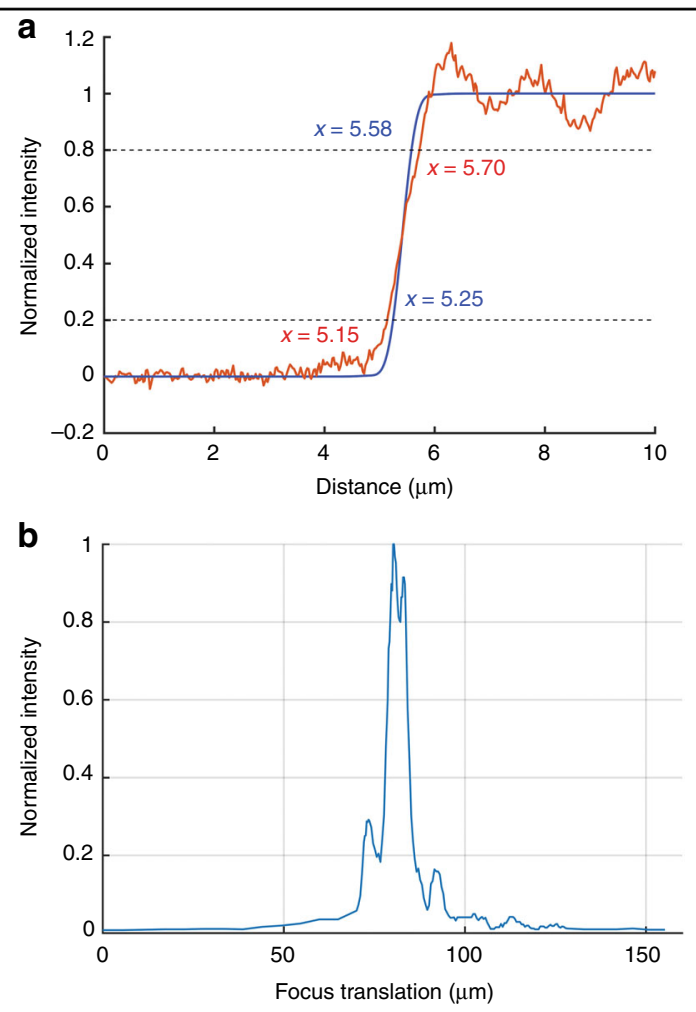

Fig. 8 Lateral and axial resolution of bench-top system. a The edge response of the confocal system with $10 \mu \mathrm{m}$ pinhole. The theoretical edge response is provided for comparison (blue). $\mathbf{b}$ The axial response of the optical system with $10 \mu \mathrm{m}$ pinhole

edge near the imaging depth at which the spherical aberration is optimally compensated by the fixed optics. Figure 8a shows a plot of the intensity data as the beam was scanned across the edge. The distance was calibrated by imaging a target of known dimensions. The measured edge width from 20 to $80 \%$ intensity is $0.55 \mu \mathrm{m}$. This can be compared with the diffraction-limited $20-80 \%$ confocal edge width of $0.33 \mu \mathrm{m}$ using NA $=0.57$. We attribute the slightly degraded response to residual manufacturing error of the MEMS mirror. For the MEMS mirror that was used for this test, we measured residual aberration, which was primarily astigmatism, with an RMS surface variation of $42 \mathrm{~nm}$ across the $4 \mathrm{~mm}$ aperture with no voltage applied. This corresponds to an RMS wavefront error of $84 \mathrm{~nm}$ in the reflected beam. This RMS wavefront error (at a wavelength of $633 \mathrm{~nm}$ ) is sufficient for degrading the Strehl ratio to $\sim 0.5$ and spreading the edge response, as we have observed.

To measure the axial response, a clean piece of silicon was mounted directly onto the sample stage (with no extra glass in the optical path). The axial focus position was adjusted by translating the MEMS mirror toward or away from the sample. For this measurement, the MEMS was acting purely as a mirror and was not deflected. The reflected light after passing through the pinhole was collected by the photodetector and the intensity was measured using an oscilloscope. Paraxial ray tracing was used to establish the relationship between the axial translation of the mirror and the axial translation of the focus point. The calibration was also verified experimentally using a microscope coverslip of known thickness $(65 \mu \mathrm{m})$ with reflective markings on both sides. The markings on either side of the coverslip were imaged by moving the focus from the front to the back of the coverslip, requiring $115 \mu \mathrm{m}$ of axial translation of the MEMS device. According to the paraxial ray trace, this equates to $63 \mu \mathrm{m}$ of focus translation in the coverslip, which closely matches the actual thickness of $65 \mu \mathrm{m}$. The calibrated axial response of the system is shown in Fig. $8 \mathrm{~b}$. The results demonstrate a full-width-at-half-maximum value of $6.1 \mu \mathrm{m}$. The theoretical, aberration-free axial response, with a finite pinhole of $10 \mu \mathrm{m}$ in diameter ${ }^{59}$, is $3.1 \mu \mathrm{m}$ full width at half maximum. Similar to the edge response, the slight broadening of the axial response is consistent with the initial 42-nm RMS surface variation, which is mostly astigmatism, of the MEMS mirror that was used for the demonstration.

\section{Acknowledgements}

This work was supported by the NIH through the National Institute of Biomedical Imaging and Bioengineering (NIBIB) (1R21EB018507) and the National Cancer Institute (NCI) (P30CA008748). The work was performed in part at the Montana Nanotechnology Facility and at the Cornell Nanoscience Facility, both members of the National Science Foundation NNCI program that is supported by NSF Grants ECCS-1542210 and ECCS-1542081. We acknowledge Martin Tauc and Ryan Downey for their assistance with the digital image acquisition.

\section{Authors' contributions}

Tianbo Liu contributed to MEMS scanner design, development, fabrication, and testing. He also constructed the benchtop confocal imaging system and performed all experimental characterizations. Milind Rajadhyaksha contributed to the concept development by working with DLD to devise the architecture for the miniature confocal imaging system. He also contributed expertise regarding clinical imaging applications. DLD contributed to the concept development, including the optical system design and simulations and the MEMS scanner specification and design. He provided overall supervision of this research effort.

\section{Conflict of interest}

The authors declare that they have no conflict of interest.

Supplementary Information is available for this paper at https://doi.org/ 10.1038/s41377-019-0167-5.

Received: 5 December 2018 Revised: 24 May 2019 Accepted: 30 May 2019 Published online: 26 June 2019

\section{References}

1. Tomita, Y. et al. Long-term in vivo investigation of mouse cerebral microcirculation by fluorescence confocal microscopy in the area of focal ischemia. J. Cereb. Blood Flow. Metab. 25, 858-867 (2005).

2. Kerr, J. N. \& Denk, W. Imaging in vivo: watching the brain in action. Nat. Rev. Neurosci. 9, 195-205 (2008).

3. Horton, N. G. et al. In vivo three-photon microscopy of subcortical structures within an intact mouse brain. Nat. Photonics 7, 205-209 (2013). 
4. Dombeck, D. A. et al. Imaging large-scale neural activity with cellular resolution in awake, mobile mice. Neuron 56, 43-57 (2007).

5. Kobat, D., Horton, N. G. \& Xu, C. In vivo two-photon microscopy to 1.6-mm depth in mouse cortex. J. Biomed. Opt. 16, 106014 (2011).

6. Davalos, D. et al. Stable in vivo imaging of densely populated glia, axons and blood vessels in the mouse spinal cord using two-photon microscopy. J. Neurosci. Methods 169, 1-7 (2008).

7. König, K. et al. Optical skin biopsies by clinical CARS and multiphoton fluorescence/SHG tomography. Laser Phys. Lett. 8, 465-468 (2011).

8. Ulrich, M. \& Lange-Asschenfeldt, S. In vivo confocal microscopy in dermatology: from research to clinical application. J. Biomed. Opt. 18, 061212 (2013).

9. Guitera, P. et al. In vivo reflectance confocal microscopy enhances secondary evaluation of melanocytic lesions. J. Invest. Dermatol. 129, 131-138 (2009).

10. Guitera, P. et al. In vivo confocal microscopy for diagnosis of melanoma and basal cell carcinoma using a two-step method: analysis of 710 consecutive clinically equivocal cases. J. Invest. Dermatol. 132, 2386-2394 (2012).

11. Nori, S. et al. Sensitivity and specificity of reflectance-mode confocal microscopy for in vivo diagnosis of basal cell carcinoma: a multicenter study. J. Am. Acad. Dermatol. 51, 923-930 (2004).

12. Rajadhyaksha, M. et al. In vivo confocal scanning laser microscopy of human skin II: advances in instrumentation and comparison with histology. J. Invest. Dermatol. 113, 293-303 (1999).

13. Zong, W. et al. Fast high-resolution miniature two-photon microscopy for brain imaging in freely behaving mice. Nat. Methods 14, 713-719 (2017).

14. Piyawattanametha, W. et al. In vivo brain imaging using a portable $2.9 \mathrm{~g}$ twophoton microscope based on a microelectromechanical systems scanning mirror. Opt. Lett. 34, 2309-2311 (2009).

15. Liu, L. et al. MEMS-based 3D confocal scanning microendoscope using MEMS scanners for both lateral and axial scan. Sens. Actuators A Phys. 215, 89-95 (2014).

16. Fu, L. et al. Nonlinear optical endoscopy based on a double-clad photonic crystal fiber and a MEMS mirror. Opt. Express 14, 1027-1032 (2006).

17. Gilchrist, K. H. et al. Piezoelectric scanning mirrors for endoscopic optical coherence tomography. J. Micromech. Microeng. 19, 095012 (2009).

18. Piyawattanametha, W. \& Wang, T. D. MEMS-based dual-axes confocal microendoscopy. IEEE J. Sel. Top. Quantum Electron. 16, 804-814 (2010).

19. Wang, Y. et al. Portable oral cancer detection using a miniature confocal imaging probe with a large field of view. J. Micromech. Microeng. 22, 065001 (2012).

20. Liu, J. T. et al. Miniature near-infrared dual-axes confocal microscope utilizing a two-dimensional microelectromechanical systems scanner. Opt. Lett. 32, 256-258 (2007).

21. Li, H. et al. Integrated monolithic 3D MEMS scanner for switchable real time vertical/horizontal cross-sectional imaging. Opt. Express 24, 2145-2155 (2016).

22. Duan, $X$. et al. Three-dimensional side-view endomicroscope for tracking individual cells in vivo. Biomed. Opt. Express 8, 5533-5545 (2017).

23. Jia, K., Samuelson, S. R. \& Xie, H. K. High-fill-factor micromirror array with hidden bimorph actuators and tip-tilt-piston capability. J. Microelectromech. Syst. 20, 573-582 (2011).

24. Liao, W. et al. A tip-tilt-piston micromirror with symmetrical lateral-shift-free piezoelectric actuators. IEEE Sens. J. 13, 2873-2881 (2013).

25. Shao, Y., Dickensheets, D. L. \& Himmer, P. 3-D MOEMS mirror for laser beam pointing and focus control. IEEE J. Sel. Top. Quantum Electron. 10, 528-535 (2004).

26. Strathman, M. et al. Dynamic focus-tracking MEMS scanning micromirror with low actuation voltages for endoscopic imaging. Opt. Express 21, 23934-23941 (2013).

27. Liu, T. et al. MEMS 3-D scan mirror with SU-8 membrane and flexures for high NA microscopy. J. Microelectromech. Syst. 27, 719-729 (2018).

28. Morrison, J. et al. Electrothermally actuated tip-tilt-piston micromirror with integrated varifocal capability. Opt. Express 23, 9555-9566 (2015).

29. Shin, H. J. et al. Fiber-optic confocal microscope using a MEMS scanner and miniature objective lens. Opt. Express 15, 9113-9122 (2007).

30. Maitland, K. C. et al. Single fiber confocal microscope with a two-axis gimbaled MEMS scanner for cellular imaging. Opt. Express 14, 8604-8612 (2006).

31. Arrasmith, C. L., Dickensheets, D. L. \& Mahadevan-Jansen, A. MEMS-based handheld confocal microscope for in-vivo skin imaging. Opt. Express 18, 3805-3819 (2010).
32. Kumar, K. Hoshino, K. \& Zhang, X. Handheld subcellular-resolution single-fiber confocal microscope using high-reflectivity two-axis vertical combdrive silicon microscanner. Biomed. Microdevices 10, 653-660 (2008).

33. Murakami, K. A miniature confocal optical scanning microscope for endoscopes. Proceedings of SPIE 5721, MOEMS Display and Imaging Systems III. San Jose, California, United States: SPIE, 2005.

34. Bechtel, C. et al. Large field of view MEMS-based confocal laser scanning microscope for fluorescence imaging. Optik 125, 876-882 (2014).

35. Chen, S. L. et al. A fiber-optic system for dual-modality photoacoustic microscopy and confocal fluorescence microscopy using miniature components. Photoacoustics 1, 30-35 (2013).

36. Lu, C. D. et al. Handheld ultrahigh speed swept source optical coherence tomography instrument using a MEMS scanning mirror. Biomed. Opt. Express 5, 293-311 (2014).

37. Duan, $X$. et al. MEMS-based multiphoton endomicroscope for repetitive imaging of mouse colon. Biomed. Opt. Express 6, 3074-3083 (2015).

38. Rouse, A. R. et al. Design and demonstration of a miniature catheter for a confocal microendoscope. Appl. Opt. 43, 5763-5771 (2004).

39. Liang, $\mathrm{C}$. et al. Design of a high-numerical-aperture miniature microscope objective for an endoscopic fiber confocal reflectance microscope. Appl. Opt. 41, 4603-4610 (2002).

40. Kester, R. T. et al. High numerical aperture microendoscope objective for a fiber confocal reflectance microscope. Opt. Express 15, 2409-2420 (2007).

41. Sun, J. et al. 3D in vivo optical coherence tomography based on a low-voltage, large-scan-range 2D MEMS mirror. Opt. Express 18, 12065-12075 (2010).

42. Jung, W. et al. Three-dimensional endoscopic optical coherence tomography by use of a two-axis microelectromechanical scanning mirror. Appl. Phys. Lett. 88, 163901 (2006).

43. Aguirre, A. D. et al. Two-axis MEMS scanning catheter for ultrahigh resolution three-dimensional and En face imaging. Opt. Express 15, 2445-2453 (2007).

44. $\mathrm{Mu}, \mathrm{X}$. et al. MEMS micromirror integrated endoscopic probe for optical coherence tomography bioimaging. Sens. Actuators A Phys 168, 202-212 (2011).

45. Wang, T. D. et al. Dual-axes confocal microscopy with post-objective scanning and low-coherence heterodyne detection. Opt. Lett. $\mathbf{2 8}$, 1915-1917 (2003).

46. Wang, T. D. et al. Dual-axis confocal microscope for high-resolution in vivo imaging. Opt. Lett. 28, 414-416 (2003).

47. Rajadhyaksha, M., Anderson, R. R. \& Webb, R. H. Video-rate confocal scanning laser microscope for imaging human tissues in vivo. Appl. Opt. 38, 2105-2115 (1999).

48. $\mathrm{Ra}, \mathrm{H}$. et al. Three-dimensional in vivo imaging by a handheld dual-axes confocal microscope. Opt. Express 16, 7224-7232 (2008).

49. Qiu, Z. et al. Targeted vertical cross-sectional imaging with handheld nearinfrared dual axes confocal fluorescence endomicroscope. Biomed. Opt. Express 4, 322-330 (2013).

50. Born, M. \& Wolf, E. Principles of Optics: Electromagnetic Theory of Propagation, Interference and Diffraction of Light. 6th edn. (Amsterdam: Elsevier, 2013).

51. Knüttel, A. \& Boehlau-Godau, M. Spatially confined and temporally resolved refractive index and scattering evaluation in human skin performed with optical coherence tomography. J. Biomed. Opt. 5, 83-92 (2000).

52. Bashkatov, A. N., Genina, E. A. \& Tuchin, V. V. Optical properties of skin, subcutaneous, and muscle tissues: a review. J. Innov. Opt. Health Sci. 4, 9-38 (2011).

53. Urey, $H$. Torsional MEMS scanner design for high-resolution scanning display systems. Opt. Scanning 4773, 27-38 (2002).

54. Lukes, S. J. \& Dickensheets, D. L. SU-8 2002 surface micromachined deformable membrane mirrors. J. Microelectromech. Syst. 22, 94-106 (2013).

55. Sung, $K$. et al. Near real time in vivo fibre optic confocal microscopy: subcellular structure resolved. J. Microsc. 207, 137-145 (2002).

56. Drezek, R. A. et al. Laser scanning confocal microscopy of cenvical tissue before and after application of acetic acid. Am. J. Obstet. Gynecol. 182, 1135-1139 (2000).

57. Liu, T. \& Dickensheets, D. L. 3-Dimensional beam scanner for a handheld confocal dermoscope. 2016 International Conference on Optical MEMS and Nanophotonics. Singapore, (IEEE).

58. Lukes, S. J. et al. Four-zone varifocus mirrors with adaptive control of primary and higher-order spherical aberration. Appl. Opt. 55, 5208-5218 (2016).

59. Wilson, T. \& Carlini, A. R. Size of the detector in confocal imaging systems. Opt. Lett. 12, 227-229 (1987). 